\title{
Callosal morphology in schizophrenia: what can shape tell us about function and illness? ${ }^{\dagger}$
}

\author{
Mark Walterfang and Dennis Velakoulis
}

\section{Summary}

Examination of the corpus callosum provides a window to cortical brain change in brain disorders. Combining volumetric with microstructural analysis allows a greater understanding of the biology underpinning change, and examining callosal structure alongside the structure of the cortical regions it interconnects may allow us to understand the true significance of callosal change in psychiatric disorders.

\section{Declaration of interest}

None.
Mark Walterfang (pictured) is a consultant neuropsychiatrist at the Neuropsychiatry Unit at Royal Melbourne Hospital and an Associate Professor of Neuropsychiatry at the Melbourne Neuropsychiatry Centre. Dennis Velakoulis is Clinical Director at the Neuropsychiatry Unit at Royal Melbourne Hospital and a Professor of Neuropsychiatry in the Melbourne Neuropsychiatry Centre.

'In so far as the statements of geometry speak about reality, they are not certain; and in so far as they are certain, they do not speak about reality.'

Albert Einstein

The corpus callosum (Latin: 'tough body') is the human brain's largest white matter tract, and its largest commissure. ${ }^{1,2}$ The callosum was first named by Galen of Pergamum in ancient Rome, at the beginning of the first century $\mathrm{AD}$, among a range of other central nervous system structures. Up until the 16th century, it was generally felt to be a supporting or 'scaffolding' structure only. In 1543, Andreas Vesalius described its anatomy for the first time, recognising that it linked the two halves of the brain and was continuous with the white matter of the hemispheres. In the 1600s, La Peyronie, Professor of Surgery at Montpellier, selected it as the 'seat of the soul', as it seemed to be the most interconnected of brain structures.

Our modern view of the corpus callosum recognises its crucial role in connecting the cerebral hemispheres. The callosum forms a high-bandwidth neural pathway between the two hemispheres, facilitating information transfer and unifying information that enters in a lateralised fashion into the hemispheric system. It contains in the region of 60 million axons, ${ }^{3-5}$ fibres that mediate sensory-motor coordination, and connect equivalent association cortical regions across hemispheres. ${ }^{6}$ The callosum has a critical role in attentional arousal, and the integration and communication of high-level motor, cognitive and sensory information. ${ }^{7}$ Given that fibres from most cortical regions traverse the callosum, it is seen as an attractive structure to study as regional brain changes can be reflected in regional shape changes in the callosum.

\section{Callosal morphology in schizophrenia}

With the advent of modern neuroimaging techniques, the callosum became an attractive candidate for analysis via structural imaging: an obviously bright structure on sagittal images, it is

'See pp. 55-60, this issue. readily segmented from surrounding structures; its unique shape profile lends itself to a range of morphological descriptors, such as area, thickness and curvature. Unlike other brain structures, the callosum does not lend itself to anatomically meaningful volume estimations because of the absence of clear lateral boundaries; researchers have thus relied on estimates of area or shape. Its topographical fibre organisation - where anterior segments connect anterior cortical regions and posterior segments connect posterior cortical regions ${ }^{8}$ - suggests that regional cortical alterations may be detectable as subtle regional changes in callosal shape or thickness. In this way, the callosum potentially provides a window to cortical changes in brain disorders, and such changes have been of clinical utility in the differentiation of dementia subtypes. ${ }^{9}$ The shape of other topographically organised and projecting structures, such as the basal ganglia, has similarly been shown to differentiate between subtypes of neurodegenerative disorders. ${ }^{10}$

The first magnetic resonance imaging (MRI) study suggested alterations in corpus callosum size and shape. ${ }^{11}$ Since this initial study, the widespread availability of MRI scanning has resulted in a range of studies examining the callosum from a variety of morphological perspectives, revealing a number of ways in which the callosum in people with schizophrenia appears to differ from healthy controls, and across different stages of illness (Fig. 1). ${ }^{12-14}$ Two meta-analyses of these studies, almost 15 years apart, are consistent with the finding that the callosum is smaller in schizophrenia, ${ }^{15,16}$ although the latter meta-analysis suggests that this effect may be greatest in patients with first-episode schizophrenia. The difficulty in extracting the nature of the true signal regarding callosal morphology in schizophrenia across these studies is the significant variety of methodological approaches used (with manifold ways to account for head size, choose a consistent mid-sagittal image across participants and parcellate the callosum in anatomically meaningful ways), and the way that descriptors of the callosum are used in these studies: 'shape', 'size' and 'reduction' are terms that have different meanings according to the differences across methodological approaches and statistical models. This heterogeneity may thus dilute or alter the findings from meta-analyses that attempt to combine studies with varying methodology; thus large multistage cohorts who are examined across different phases of psychosis utilising the same sequence parameters and image analysis ${ }^{12-14}$ may be preferable.

Given that evidence suggests that brain changes may occur prior to illness onset, and may progress with transition to 

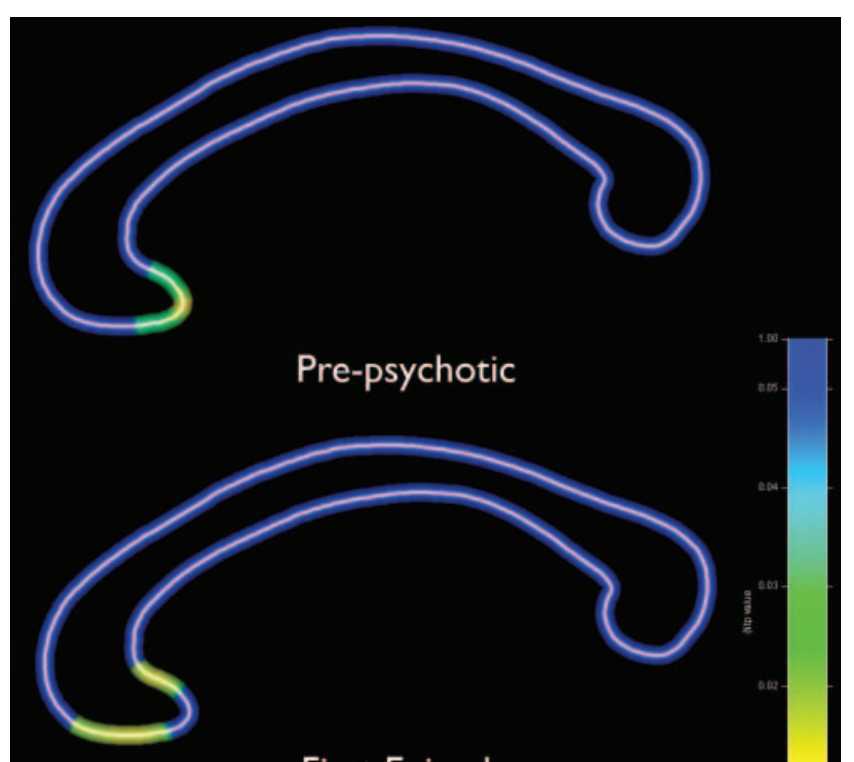

First Episode

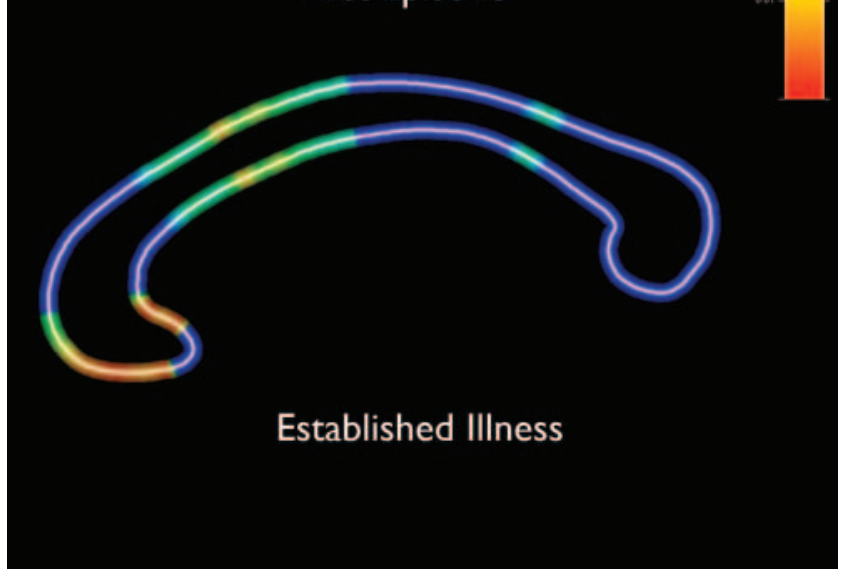

Fig. 1 Callosal significance maps of regional thickness reductions shown previously in individuals who are pre-psychotic (top), with first-episode psychosis (middle) and with established schizophrenia (bottom). ${ }^{12-14}$

Each significance map is projected onto a callosal skeleton in blue; regions of significance change are marked in colour on the skeleton. Patients who are pre-psychotic (top) show changes in the anterior genu, connecting frontal cortical regions, which are also seen in patients with first-episode psychosis (middle) across a larger region of the genu; established patients (bottom) show more significant reductions in this anterior region, but then show reductions in the body and isthmus of the callosum, connecting temporal cortical regions.

psychosis and/or established illness, a true understanding of shape changes as described by any particular method would be applied across different stages of illness: pre-psychotic, first-episode and established illness. ${ }^{12,13}$ Additionally, volumetric imaging - from which callosal shape can be derived - is limited in its capacity to inform on microstructural changes within brain regions. Recent white matter imaging techniques such as diffusion tensor imaging (DTI) suggest that some of the callosal shape alterations in schizophrenia are driven by alterations to white matter microstructure, either through disrupted myelination or altered axonal structure or organisation. ${ }^{17}$ Finally, although white and grey matter structures are arbitrarily separated based on their macrostructural appearance and intensity on $T_{1}$-weighted MRI sequences, they remain different components of groups of the same functional unit: the neuron and its axonal process. When white matter change is seen in any illness, the interdependent nature of grey and white matter compartments means that change may be driven by a mixture of primary white matter pathology, or alterations that occur secondary to grey matter change. ${ }^{18,19}$ As a result, a true determination of the role of white matter change in any cohort of patients with schizophrenia will necessarily also analyse grey matter findings in the same cohort.

The study described by Collinson et al in this issue addresses some, albeit not all, of these issues within one large cohort of 120 patients (with first-episode and established schizophrenia) and 75 controls. ${ }^{20}$ This study utilised both high-resolution volumetric $T_{1}$-weighted images and diffusion-tensor images acquired in the same session on all participants. This welldesigned study has some compelling advantages: a large sample size recruited from one geographical region; patients at different illness stages; and an imaging protocol examining both volumetric and microstructural measures of the callosum. It showed that the differences were detectable only between patients with established schizophrenia and controls, but individuals with first-episode schizophrenia showed no significant difference. Additionally, the DTI measures, which could be expected to potentially underpin volumetric change, showed no difference on any group analysis. It adds to the heterogeneity of findings in patients with schizophrenia: the most recent structural imaging meta-analysis suggests that volumetric reductions may be greatest in first-episode rather than established illness, ${ }^{16}$ and a meta-analysis of DTI findings suggests significant reductions in microstructural integrity of the splenium across studies. ${ }^{21}$ The Collinson et al study also illustrates some of the potential pitfalls of studying any structure in schizophrenia, where the choice of methodology may have an impact on the capacity to detect change. One key example is in accounting for head size; many callosal studies have covaried for intracranial volume to account for this, although using a linear transformation and scaling has been shown to be superior in detecting true between-group differences; ${ }^{22}$ transformation is part of the FreeSurfer pipeline used by Collinson and colleagues in their analysis. Furthermore, most studies of the callosum attempt to find local regional differences, thus moving from a pure area/ volume measure to a de facto metric of shape. Choice of shape metric and method may similarly affect the capacity to detect results, with sophisticated, sensitive and anatomically informed shape analysis methods for the callosum now being described. ${ }^{23,24}$ A move to these more sophisticated methods, combined with the microstructural methods as utilised in the Collinson et al study, are - at least in part - the way to move the field forward.

\section{Callosum and corollary discharges}

How can an understanding of changes in the callosum inform us about the neurobiology of schizophrenia? As the brain's largest white matter fibre bundle, if there are diffuse white matter changes in schizophrenia, callosal interhemispheric fibres - alongside long association tracts - are most likely to have both their structure and function disrupted. Consequent abnormalities in neural timing and synchrony between distal brain regions may thus produce the characteristic symptoms of schizophrenia. One particular function is highly dependent on accurate timing in these distal brain regions: corollary discharges, which are neural signals that are initiated in frontal cortical regions coincident with willed actions and fed posteriorly to sensory regions to suppress the sensory consequences of these actions, thereby allowing the brain to recognise these actions as 'self'-generated. ${ }^{25}$ With subtle changes to white matter integrity, an introduced delay in this system may result in 'self'-generated phenomena being erroneously experienced as 'other': internal speech becomes 
external auditory hallucinations, internal images or thoughts are experienced as projected from an external source and somatic experiences as passivity phenomena. ${ }^{26}$ Callosal microstructural abnormalities in schizophrenia are known to be accompanied by abnormal neural timing ${ }^{27}$ and correlate with the severity of these psychotic symptoms. ${ }^{28}$ Thus, alterations in the callosal macroand microstructure may be an index in people with schizophrenia of the severity of neural timing abnormalities, and thus the likelihood of the pathognomonic reality distortions in these patients whereby the distinction between 'self' and 'other' breaks down during episodes of psychosis. The Collinson et al ${ }^{20}$ findings do not completely align with this hypothesis however, as changes would be expected to be detectable during both first-episode and established illness, as individuals are likely to experience these symptoms across illness stages. As the role of the callosum in schizophrenia pathology remains far from settled, the dissonance between the findings in the Collinson et al study and the corollary discharge hypothesis, and other models of schizophrenia that focus on the role of white matter, ${ }^{18,19}$ is likely to remain irresolvable for some time.

\section{Conclusion}

Magnetic resonance imaging in psychiatric illness provides rich data-sets that allow for a wide range of investigative approaches. The callosum is an attractive candidate for neuroimaging analysis as it is readily segmented, can be analysed in two as well as three dimensions, and has shape characteristics that can be measured manifold. Additionally, its degree of connectedness with disparate brain regions means that it can be used as an index for both white and grey matter change in the brain. However, the increasing variegation in analytical approaches to callosal structure may magnify, rather than simplify, the heterogeneity of complex mental disorders such as schizophrenia. Making future callosal studies meaningful in the context of schizophrenia requires us to move into more whole-of-brain, whole-of-illness approaches, which tie both structure and function together. This may allow us to determine whether the callosum in schizophrenia is the 'seat' of the illness, or merely its reflector.

Mark Walterfang, MBBS(Hons), PhD, FRANZCP, Dennis Velakoulis, MBBS, MMed, DMedSci, FRANZCP, Neuropsychiatry Unit, Royal Melbourne Hospital and Melbourne Neuropsychiatry Centre, University of Melbourne and Melbourne Health, Melbourne Australia

Correspondence: Mark Walterfang, Level 2, John Cade Building Royal Melbourne Hospital, Parkville, Victoria 3050, Australia. Email: mark.walterfang@mh.org.au

First received 20 Jul 2013, final revision 23 Sep 2013, accepted 10 Oct 2013

\section{References}

1 Innocenti G, Bressoud R. Callosal axons and their development. In The Parallel Brain: The Cognitive Neuroscience of the Corpus Callosum (eds E Zaidel, M Iacoboni): 11-26. MIT Press, 2003.

2 Schmahmann J, Pandya D. Corpus callosum. In Fiber Pathways of the Brain (eds J Schmahmann, D Pandya): 485-96. Oxford University Press, 2006.

3 LaMantia A, Rakic P. Axon overproduction and elimination in the corpus callosum of the developing rhesus monkey. J Neurosci 1992; 10: 2156-75.

4 Aboitiz F, Scheibel A, Fisher R, Zaidel E. Fiber composition of the human corpus callosum. Brain Res 1992; 598: 143-53.

5 Aboitiz F, Scheibel A, Fisher R, Zaidel E. Individual differences in brain asymmetries and fiber composition in the human corpus callosum. Brain Res 1992: 598: 154-61.
6 Yazgan M, Kinsbourne M (2003) Functional consequences of changes in callosal area in Tourette's syndrome and attention deficit/hyperactivity disorder. In The Parallel Brain: The Cognitive Neuroscience of the Corpus Callosum (eds E Zaidel, M lacoboni): 423-32. MIT Press, 2003.

7 Hoptman MJ, Davidson RJ. How and why do the two cerebral hemispheres interact? Psychol Bull 1994; 116: 195-219.

8 Pandya D, Seltzer B. The topography of commisural fibers. In Two Hemispheres, One Brain Functions of the Human Corpus Callosum (eds F Lepore, M Pitto, H Jasper): 47-73. Alan R Liss, 1986.

9 Yamauchi $\mathrm{H}$, Fukuyama $\mathrm{H}$, Nagahama $\mathrm{Y}$, Katsumi $\mathrm{Y}$, Hayashi T, Oyanagi C, et al. Comparison of the pattern of atrophy of the corpus callosum in frontotemporal dementia, progressive supranuclear palsy, and Alzheimer's disease. J Neurol Neurosurg Psychiatry 2000; 69: 623-9.

10 Looi JC, Walterfang M. Striatal morphology as a biomarker in neurodegenerative disease. Mol Psychiatry 2013; 18: 417-24.

11 Nasrallah H, Andreasen N, Coffman J, Olson S, Dunn V, Ehrhardt JC, et al. A controlled magnetic resonance imaging study of corpus callosum thickness in schizophrenia. Biol Psychiatry 1986; 21: 274-82.

12 Walterfang $M$, Wood AG, Reutens DC, Wood SJ, Chen J, Velakoulis D, et al Morphology of the corpus callosum at different stages of schizophrenia: cross-sectional study in first-episode and chronic illness. Br J Psychiatry 2008; 192: 429-34.

13 Walterfang M, Yung A, Wood A, Reutens D, Phillips L, Woods SJK, et al. Corpus callosum shape alterations in individuals prior to the onset of psychosis. Schizophr Res 2008; 103: 1-10.

14 Walterfang M, Wood AG, Reutens DC, Wood SJ, Chen J, Velakoulis D, et al. Corpus callosum size and shape in first-episode affective and schizophreniaspectrum psychosis. Psychiatry Res 2009; 173: 77-82.

15 Woodruff $P$, McManus I, David A. Meta-analysis of corpus callosum size in schizophrenia. J Neurol Neurosurg Psychiatr 1995; 58: 457-61.

16 Arnone D, McIntosh A, Tan G, Ebmeier K. Meta-analysis of magnetic resonance imaging studies of the corpus callosum in schizophrenia. Schizophr Res 2008; 101: 124-32.

17 Yao L, Lui S, Liao Y, Du MY, Hu N, Thomas JA, et al. White matter deficits in first episode schizophrenia: an activation likelihood estimation meta-analysis. Prog Neuropsychopharmacol Biol Psychiatry 2013; 45C: 100-6.

18 Walterfang $M$, Wood S, Velakoulis D, Pantelis C. Neuropathological, neurogenetic and neuroimaging evidence for white matter pathology in schizophrenia. Neurosci Biobehav Rev 2006; 30: 918-48.

19 Walterfang M, Velakoulis D, Whitford TJ, Pantelis C. Understanding aberrant white matter development in schizophrenia: an avenue for therapy? Expert Rev Neurother 2011; 11: 971-87.

20 Collinson SL, Gan SC, Woon PS, Kuswanto C, Sum MY, Yang GL, et al. Corpus callosum morphology in first-episode and chronic schizophrenia: combined magnetic resonance and diffusion tensor imaging study of Chinese Singaporean patients. Br J Psychiatry 2014; 204: 55-60.

21 Patel S, Mahon K, Wellington R, Zhang J, Chaplin W, Szeszko PR. A meta-analysis of diffusion tensor imaging studies of the corpus callosum in schizophrenia. Schizophr Res 2011; 129: 149-55.

22 Bermudez P, Zatorre RJ. Sexual dimorphism in the corpus callosum: methodological considerations in MRI morphometry. Neuroimage 2001; 13: $1121-30$.

23 Herron TJ, Kang X, Woods DL. Automated measurement of the human corpus callosum using MRI. Front Neuroinform 2012; 6: 25.

24 Adamson $\mathrm{CL}$, Wood AG, Chen J, Barton S, Reutens DC, Pantelis C, et al. Thickness profile generation for the corpus callosum using Laplace's equation. Hum Brain Mapp 2011; 32: 2131-40.

25 Crapse TB, Sommer MA. Corollary discharge circuits in the primate brain. Curr Opin Neurobiol 2008; 18: 552-7.

26 Whitford TJ, Ford JM, Mathalon DH, Kubicki M, Shenton ME. Schizophrenia, myelination, and delayed corollary discharges: a hypothesis. Schizophr Bull 2012; 38: 486-94

27 Whitford TJ, Kubicki M, Ghorashi S, Schneiderman JS, Hawley KJ, McCarley RW, et al. Predicting inter-hemispheric transfer time from the diffusion properties of the corpus callosum in healthy individuals and schizophrenia patients: a combined ERP and DTI study. Neuroimage 2011; 54: 2318-29.

28 Whitford TJ, Kubicki M, Schneiderman JS, O’Donnell LJ, King R, Alvarado JL, et al. Corpus callosum abnormalities and their association with psychotic symptoms in patients with schizophrenia. Biol Psychiatry 2010; 68: 70-7. 